\title{
Correlation between Sum of 8 Skinfolds to Predicted \% Body Fat Range as a Reliable Measure of Body Composition Assessment for Well-Trained Athletes
}

\author{
Ballard R. J. ${ }^{1,3}$, Dewanti R. A. ${ }^{1,2}$, Sayuti S. ${ }^{1,3}$ \& Umar N. ${ }^{1,3}$ \\ ${ }^{1}$ Sport Science Department, KONI (Komite Olahraga Nasional Indonesia) Provinsi Sumatera Barat, Indonesia \\ ${ }^{2}$ Faculty of Sports Sciences, Universitas Negeri Jakarta, Jakarta, Indonesia \\ ${ }^{3}$ Faculty of Sports Sciences, Universitas Negari Padang, Sumatera Barat, Indonesia \\ Correspondence: Ballard R. J., Faculty of Sports Sciences, Universitas Negeri Padang, Jl. Prof. Dr. Hamka - Air \\ Tawar, Padang, Sumatera Barat, Indonesia. E-mail: robertjohn_ballard@y7mail.com
}

Received: January 16, 2014 Accepted: February 20, 2014 Online Published: February 28, 2014

doi:10.5539/ass.v10n5p12 URL: http://dx.doi.org/10.5539/ass.v10n5p12

\begin{abstract}
The purpose of this study was to examine if relationship trends between the mean of 3 predicted $\%$ body fat $(\% \mathrm{BF})$ equations and the sum of 8 skinfold (SKF) measures existed for well-trained athletes as opposed to BMI as an assessment of body composition. Two test periods were conducted 4 months apart collecting 8 SKF measures from 35 full-time athletes ( 21 male $26.05 \pm 5.07$ and 14 female $24 \pm 4.15)$, 17 athletes were tested at both periods. Predicted \%BF calculation used was the mean score (MS) of three equation predictors - Durnin and Womersley (DW) 4 Site Skinfold Test (Standard Error of Estimate (SEE) 3.5\% for female: $4.0 \%$ for male), Jackson and Pollock (JP) 3 site SKF Test (SEE 3.9\% for female: 3.4\% for male), and Yuhasz SKF Test (Total Error (TE) 3.5\% for male). Easton et al (1995) indicated JP \& DW over and underestimated \%BF, which suggests the mean may reduce the error of calculation. This analysis did support Easton et al observations with mean results for male $8.71 \pm 5.11(16.01 \%<\mathrm{MS}) \mathrm{JP}, 12.80 \pm 5.56 \mathrm{DW}(23.36 \%>\mathrm{MS}), 9.61 \pm 3.16$ Yuhasz and MS of $10.37 \pm 4.55$; female $17.41 \pm 6.22(4.08 \%<\mathrm{MS}) \mathrm{JP}, 23.86 \pm 6.67(31.42 \%>\mathrm{MS}) \mathrm{DW}, 13.19 \pm 7.50$ Yuhasz and MS $18.15 \pm 6.72$. The correlation co-efficient relationships for all equations were significant from $r=0.965$ to $r=$ 0.983 for males and $r=0.961$ to $r=0.992$ for female. The MS data indicated range trends to link the sum of 8 SKF measures to a prediction range of $\% \mathrm{BF}$. However the data sample is small in determining definitive conclusions, this warrants further data collection to validate the range trend findings. The data from the 17 subjects highlighted BMI has deficiencies in determining a true reflection of a well-trained athlete's body composition.
\end{abstract}

Keywords: body composition, skinfold measurements, athletes

\section{Introduction}

Many coaches, athletes and sporting committee boards are still keen on the \%BF of an athlete and not satisfied with the sum of skinfolds or BMI indicators. So the purpose of this analysis was to determine if there are suitable range scores in the sum of $8 \mathrm{SKF}$ measures that link to a $\% \mathrm{BF}$ range score to access a more consistent prediction of body composition for athletes and to determine if BMI method is an appropriate assessment indicator of body composition for athletes.

The BMI was invented by Adolphe Quetlet in the mid $19^{\text {th }}$ Century. Although the index does not measure the percentage of body fat, it is used to estimate a healthy body weight based on a person's height. It is the most widely used metric for identifying individuals with weight problems within a population due to its ease of measurement and calculation (Note 1). BMI was originally called the Quetelet Index until it was termed the Body Mass Index in 1972 by Ancel Keys, for his application of comparative statistics to social conditions and moral issues (Note 2). A focus of this analysis was to determine if BMI was a reliable measurement of body composition for well-trained athletes. The digital pictorials (Note $2 \& 3$ ) of male and female as shown in digital diagrams 1 and 2 detail the BMI guidelines of body weight and height scores $\left(\mathrm{Kg} / \mathrm{m}^{2}\right)$, range terminology and pictorial progressions <17.5 Anorexia, 17.6 - 18.5 Underweight, 18.6 - 22 Lowest Normal, 22.1 - 24.9 Middle Normal, 25 -30 Overweight Obesity, >30 Overweight Morbid Obesity. BMI is certainly the easiest and most 
basic of measurements of estimating body composition however BMI does not take into account the difference in lean body mass (LBM) such as a well-trained athlete may possess. BMI is calculated on Mass and Height $\left(\mathrm{Mass} / \mathrm{m}^{2}\right.$ ) whereas anthropometric (skinfold) measurement methods are more specific in estimating LBM through a regression equation. Such as the SKF measurements developed for adult males and females by Jackson and Pollock (1978) in which there are other such equations of skinfolds for determining \%BF all of which are reported to be more reliable than the BMI method and can be completed out of a laboratory relatively easily (Note 4). SKF use for the Prediction \%BF has been researched and debated significantly for decades. While modern technological advances with controlled high tech methods conducted under laboratory conditions producing higher reliability and more accurate prediction of $\% \mathrm{BF}$ is available such as DEXA (Dual energy $\mathrm{x}$-ray absorptiometry) and Hydrodensitometry (underwater weighing) methods. Unfortunately much of this technology is out of reach for a majority of athletes and coaches, be it a cost factor or availability to such technologies. This analysis attempted to find a way to minimize the error of SKF \% calculations by using the MEAN SCORE (MS) of current researched and documented protocol SKF equations Jackson and Pollock, Durnin and Womersley, and Yuhasz methods. Understanding each one has variations in SEE (standard error of estimate) and TE (total error) for either over or underestimating \% $\mathrm{BF}$, as supported by Eston RG et al (1995) stating, "It is concluded that the Durnin and Womersley and Jackson and Pollock equations tend to overestimate and underestimate percentage fat, respectively, when compared with hydrodensitometry" and "The equation of Durnin and Womersley accuracy (standard error of estimate (SEE)) is reported to be $3.5 \%$ for women and $4.0 \%$ for men.". For reference the SEE of Jackson and Pollock (3 site) is reported to be 3.9\% for female and 3.4\% for male. TE (total error) for Yuhasz is reported to be $3.5 \%$ for men.

\section{Methodology}

Two test periods 4 months apart were conducted as per the protocols of a comprehensive physical performance measurement procedural manual (Note 5) specifically compiled and written including all protocols of a large battery of tests covering: body composition, speed, power, strength, aerobic power, core control/stabilization aligned to set target parameters to meet expected performance goals. For the purposes of this study, all SKF measures were collated and analysed from the 2 test periods to determine if any range trends were present within the sum of $8 \mathrm{SKF}$ measures compared to the predicted $\% \mathrm{BF}$ range of the subjects, additionally comparing if BMI was a reliable indicator of body composition for well-trained athletes.

The subjects for this analysis were province, national and international Indonesian sportsmen and women in full training for their National Sport Championships. A total of 35 subjects (21 male and 14 female) from a number of sporting disciplines were selected. Their training schedule consisted of 10-12 sessions per week including technical, tactical and strength training sessions, amassing 25-30 hours of training per week. 17 of the 35 subjects $(48.57 \%)$ had SKF measures recorded at both test periods to provide a sample total of 52 data assessments.

Table 1. Athlete profile characteristics

\begin{tabular}{lcccc}
\hline & \multicolumn{2}{c}{ Height } & Mass & \multicolumn{3}{c}{ Age } \\
\hline Male & $168.83 \pm 7.06$ & $67.57 \pm 11.01$ & & $26.05 \pm 5.07$ \\
Female & $154.09 \pm 4.40$ & $53.35 \pm 8.80$ & 24 & \pm 4.15 \\
\hline
\end{tabular}

All athletes prior to SKF measurement had been taken through a standardized warm up over 20 minutes consisting of easy to moderate movement, dynamic drills and stretches all completed indoors to raise body temperature in preparation for the full session of performance tests to be conducted. As per the testing procedural manual 8 SKF measures were recorded, and calculated for estimated \%BF including BMI calculations. All completed SKF measures for male and female subjects were conducted by the same tester at both testing periods and overseen by the same supervisor.

For height recording, athletes were required to be barefoot and standing straight against an already marked out height chart on a solid cement wall. The wall chart was calibrated with a metric tape measure to ensure accuracy was maintained. A slide square was use against the wall and placed on top of the athletes head at the same time and the height was recorded as displayed on the wall chart where the slide square had stopped.

Standard bathroom scales were used placed on a flat tiled floor for the recording of body weight. The scales were checked and centre after each recording, all athletes were weighed in their under garments only. 
The calipers used for the SKF measurement were the Slim Guide Caliper and used at both testing periods where 8 measures were recorded; Triceps, Subscapula, Biceps, Suprailiac, Chest (for men), Rear Thigh (for women), Abdomen, Front Thigh and Calf. All site locations were as per the procedural manual protocols and calculated into 3 predicted $\% \mathrm{BF}$ equation methods with the mean of the 3 equations recorded as the final result.

Table 2. Sites for skinfold measurement according to different test protocols

\begin{tabular}{|c|c|c|c|c|c|c|}
\hline \multicolumn{7}{|c|}{ Protocol 1: Durnin and Womersley 4 site SKF test } \\
\hline Male and Female & Triceps & Bicep & Subscapular & \multicolumn{2}{|c|}{ Suprailiac } & \\
\hline \multicolumn{7}{|c|}{ Protocol 2: Jackson and Pollock 3 site SKF Test } \\
\hline Male & Chest & Abdomen & Thigh (Front) & & & \\
\hline Female & Triceps & Suprailium & Thigh (Front) & & & \\
\hline \multicolumn{7}{|c|}{ Protocol 3: Yuhasz 3 sites SKF test } \\
\hline Male & Triceps & Subscapular & Suprailiac & Abdomen & Front Thigh & Chest \\
\hline Female & Triceps & Subscapular & Suprailiac & Abdomen & Front Thigh & Rear Thigh \\
\hline
\end{tabular}

\section{Data Analysis}

All male and female data from the 2 test periods were collated, separated and recorded. Additional data recorded were mean \pm standard deviation (STDEV) for each group of males and females. The mean of the 3 protocols was recorded as the final result of predicted \%BF (MS). BMI was calculated and recorded from the mass and height data for later comparison and analysis.

Statistical correlation co-efficient ( $r$ value) was conducted on all 3 equations and the MS for both male and female groups to determine the statistical significance of the relationship between SKF SUM: \%BF. The significance level used is set at $\alpha=0.05$ with $d f=27$ male and 21 female giving where $r=0.367$ male and $r=$ 0.413 female.

\section{Results}

Table 3 show the mean and standard deviation for skinfold measurements, body fat percentage and body mass index for male and female athletes.

Table 3. Mean and standard deviation for all variables

\begin{tabular}{lll}
\hline & Male & Female \\
\hline Sum 8 SKF & $71.03 \pm 33.97$ & $96.87 \pm 39.23$ \\
Body Fat \% MS & $10.37 \pm 4.55$ & $18.17 \pm 6.70$ \\
BMI & $23.63 \pm 2.99$ & $22.51 \pm 3.87$ \\
$n *$ & 29 & 23 \\
\hline$*_{n}=$ number of 8 SKF recordings & &
\end{tabular}

$*_{n}=$ number of 8 SKF recordings

Table 4 shows the mean for predicted \%BF using the equations for the 3 protocols and mean score.

Table 4. Predicted \%BF equation means

\begin{tabular}{lcccc}
\hline & Jackson \& Pollock & Durin \& Womersley & Yuhasz & Mean Score (MS) \\
\hline Male & $8.71 \% \pm 5.11$ & $12.80 \% \pm 5.56$ & $9.61 \% \pm 3.16$ & $10.37 \pm 4.55$ \\
Female & $17.41 \% \pm 6.22$ & $23.86 \% \pm 6.67$ & $13.19 \% \pm 7.50$ & $18.17 \pm 6.70$ \\
\hline
\end{tabular}

Male and Female means for Jackson and Pollock, and Yuhasz equations underestimated \%BF: MS by $16.01 \%$ \& $7.35 \%$ respectively while Durnin and Womersly overestimated \%BF: MS by $23.36 \%$ for males. Similarly 
Jackson and Pollock, and Yuhasz equations underestimated \%BF: MS 4.08\% \& 27.34\% respectively while Durnin and Womersly overestimated \%BF: MS by $31.42 \%$ for female (see Table 5 ), supporting the findings of Easton et al (1995).

Table $5 . \%$ of mean score

\begin{tabular}{cccc}
\hline & Jackson \& Pollock & Yuhasz & Durnin \& Womersley \\
\hline Male & $83.99 \%$ & $92.65 \%$ & $123.36 \%$ \\
\hline Female & $95.92 \%$ & $72.66 \%$ & $131.42 \%$ \\
\hline
\end{tabular}

\section{Relationship Trends}

A review of trends from the individual MS is listed in Tables 6 and 7. Note the relationship with the range of the sum of 8 SKF's aligned to a predicted \%BF range. Highlighted in each table is the mean of the SKF's and MS (mean score) predicted \%BF for both male and female falling into line with the range trends from the recorded test data.

Table 6. Summary review for women and suggested pattern range calculations

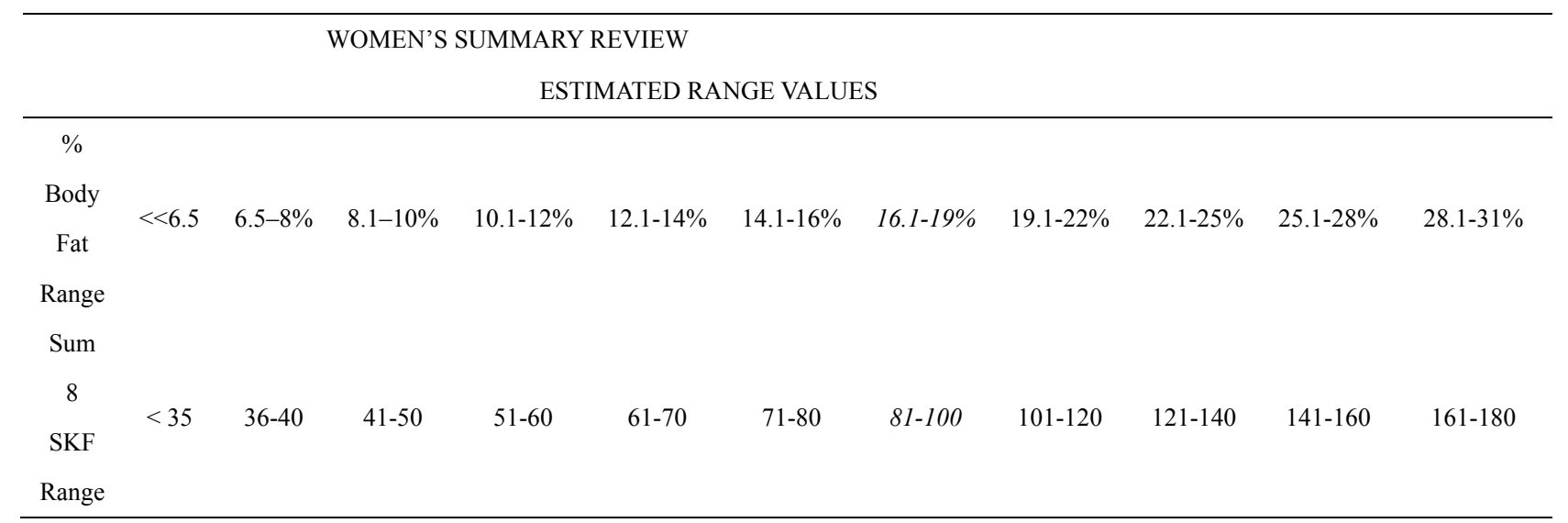

Table 7. Summary review for men and suggested pattern range calculations

\begin{tabular}{|c|c|c|c|c|c|c|c|c|c|c|c|}
\hline \multicolumn{12}{|c|}{ MENS SUMMARY REVIEW } \\
\hline $\begin{array}{c}\text { Body } \\
\text { Fat }\end{array}$ & $<4.5$ & $4.5-5.5 \%$ & $5.51-6.75 \%$ & $6.76-8 \%$ & $8.1-9.5 \%$ & $9.51-11 \%$ & $11.1-13 \%$ & $13.1-15 \%$ & $15.1-17.5 \%$ & $17.6-23 \%$ & $>23 \%$ \\
\hline Range & & & & & & & & & & & \\
\hline $\begin{array}{c}\text { Sum } \\
8 \\
\text { SKF }\end{array}$ & $<35$ & $35-40$ & $41-45$ & $46-50$ & $51-60$ & $61-75$ & $76-90$ & $91-110$ & $111-135$ & $136-160$ & $>160$ \\
\hline Range & & & & & & & & & & & \\
\hline
\end{tabular}

\subsection{BMI Score Comparison to Body Fat \% Results}

Digital diagrams $1 \& 2$ illustrate the male and female results with a cross-sectional line indicating the BMI mean. The diagrams show an extreme range of from anorexia to overweight obesity with the mean of males towards end range of middle normal and females mean of middle normal. 


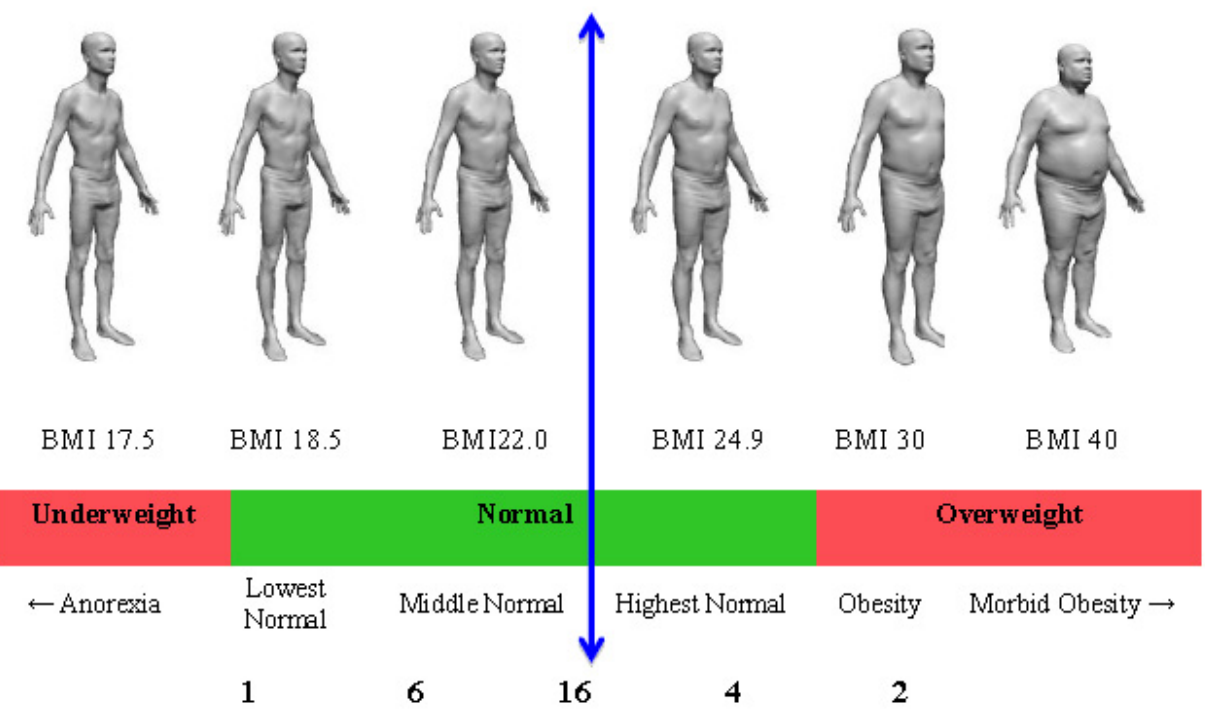

Figure 1. Male - digital diagram 1

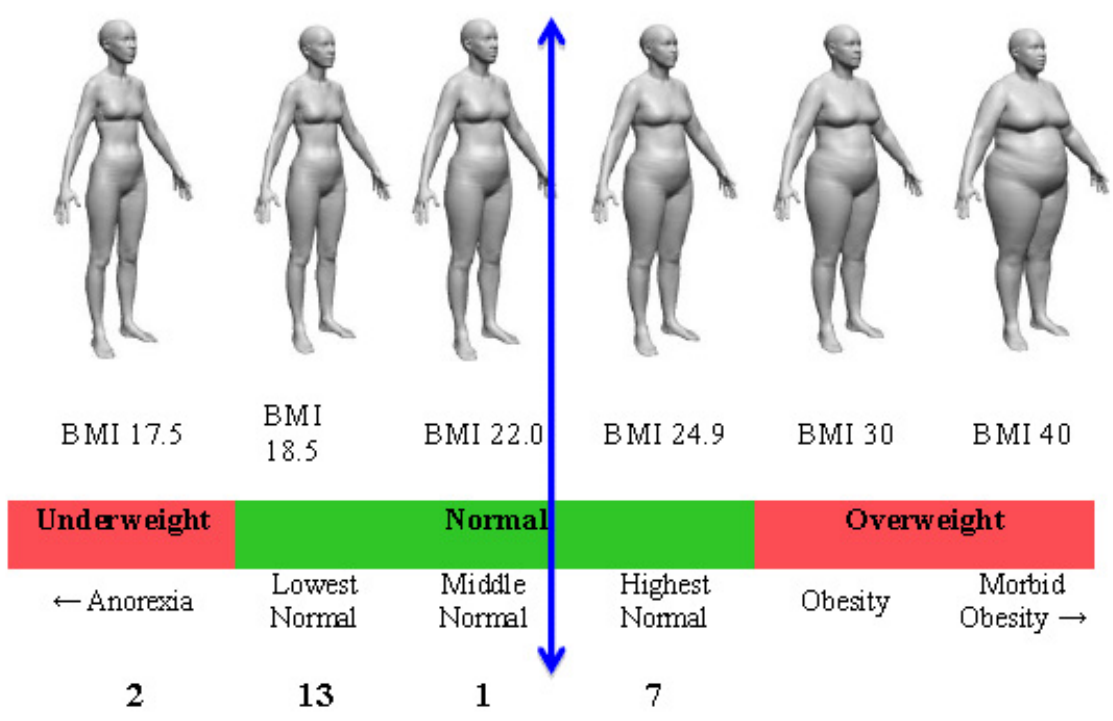

Figure 2. Female - digital diagram 2

Table 8 and Figure 3 display the result comparisons of the 17 subjects who had data recorded at both test periods. These results show there were significant changes in the subjects predicted \%BF scores while maintaining bodyweight. 
Table 8.17 athletes with 2 data recordings

\begin{tabular}{rrrrrrr}
\hline & \multicolumn{2}{c}{ July } & \multicolumn{3}{c}{ March } \\
& BF $\%$ & \multicolumn{1}{c}{ SKF } & BMI & BF \% & SKF & BMI \\
\hline \multirow{4}{*}{ MALE } & 7.50 & 49.5 & 24.77 & 8.05 & 54 & 24.44 \\
& 7.32 & 47.5 & 22.21 & 9.14 & 61 & 22.39 \\
& 13.66 & 97.5 & 30.99 & 15.91 & 118 & 32.21 \\
& 6.32 & 41.5 & 22.79 & 8.24 & 47.5 & 22.31 \\
& 8.21 & 52 & 21.39 & 9.04 & 58 & 21.39 \\
& 14.58 & 88 & 23.62 & 16.14 & 97 & 24.01 \\
& 11.42 & 82 & 22.84 & 10.15 & 69 & 22.49 \\
& 14.71 & 103 & 24.68 & 17.38 & 134 & 24.31 \\
\hline FEMALE & 8.63 & 43 & 20.45 & 9.75 & 46 & 20.45 \\
& 16.95 & 86 & 21.5 & 23.17 & 135 & 21.93 \\
& 22.15 & 123 & 25.63 & 26.29 & 144 & 26.06 \\
& 12.37 & 70 & 20.61 & 21.32 & 109 & 20.17 \\
& 21.59 & 103 & 28.83 & 29.90 & 161 & 28.83 \\
& 23.49 & 128 & 22.35 & 24.81 & 135 & 21.5 \\
AVE; & 22.49 & 127 & 29.43 & 27.17 & 156 & 28.13 \\
& 6.87 & 37 & 16.82 & 11.60 & 55 & 17.43 \\
& 10.55 & 56 & 20.61 & 15.88 & 73 & 20.61 \\
\hline
\end{tabular}

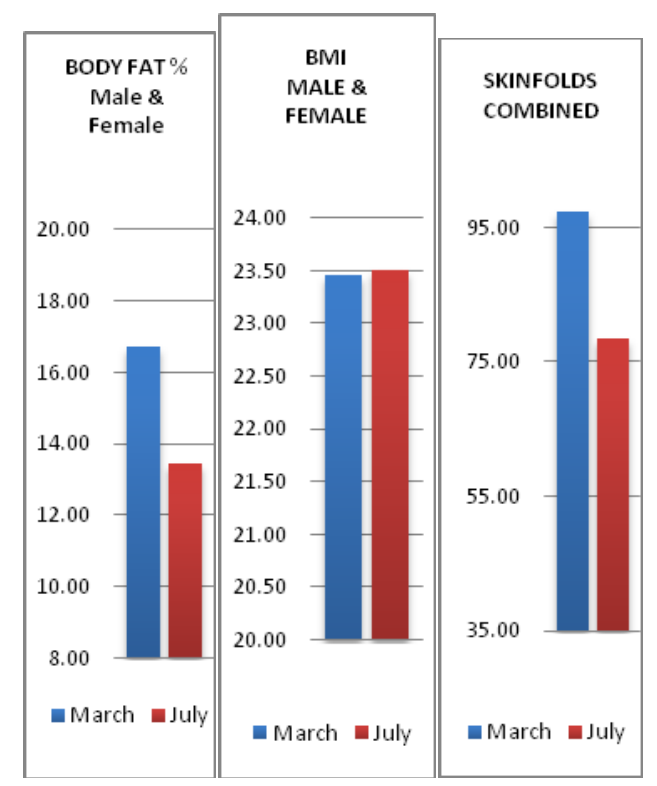

Figure 3. Displaying the comparisons of table 4

\subsection{Strength of Significance}

All the r-values of the correlation co-efficient had a very strong relationship of significance with $r=0.965$ to $r=$ 0.983 for males and $r=0.961$ to $r=0.992$ for female. It is well accepted with an increase in SKF sum will directly relate to an increase in \%BF; Refer to figures 4 - 7 for Male and 8 - 11 for Female. 
Correlations for Male SKF: \%BF

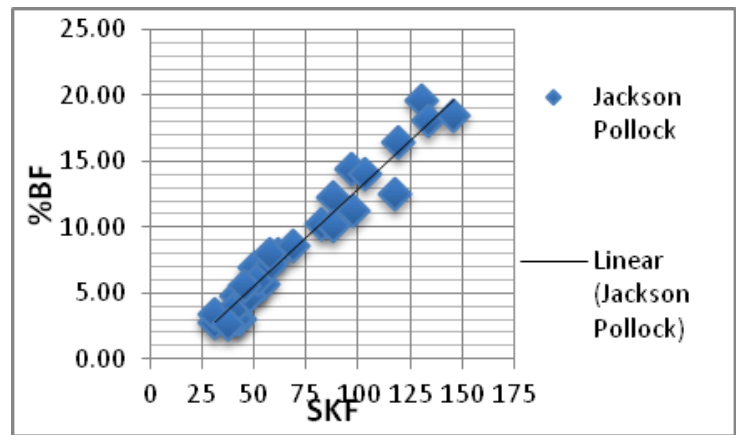

Figure 4. Jackson and Pollock $r=0.976 \mathrm{JP}$

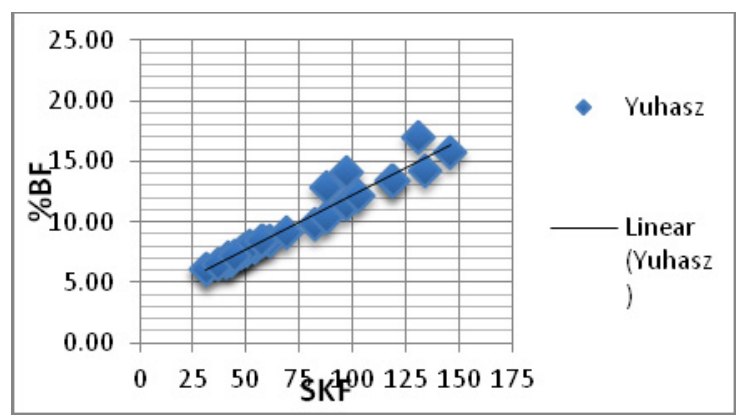

Figure 6. Yuhasz $\mathrm{r}=0.971 \mathrm{Y}$

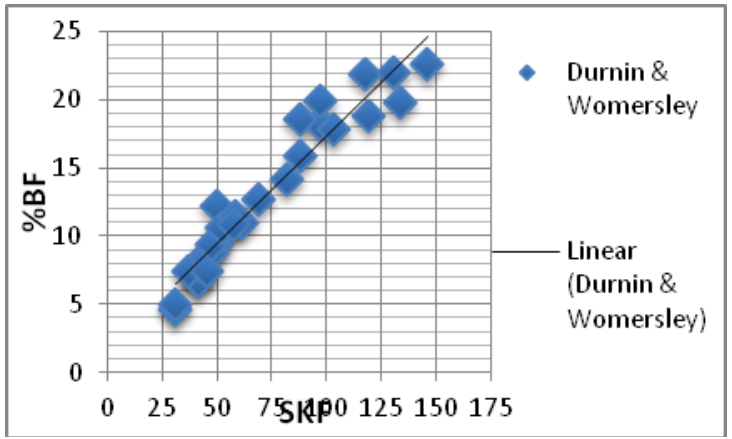

Figure 5. Durin Womersley $r=0.965 \mathrm{DW}$

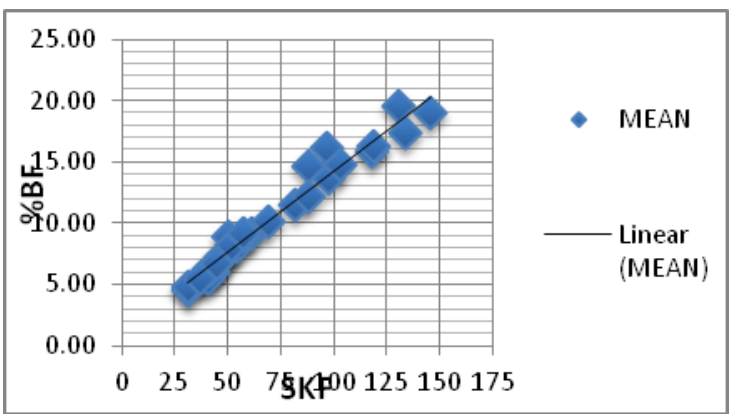

Figure 7. Mean Score $r=0.983$ MS

The MS displays the highest relationship of significance for males, acknowledging the MS has of a higher reliability relationship of significance than each individual equation.

Correlations for Female SKF: \%BF;

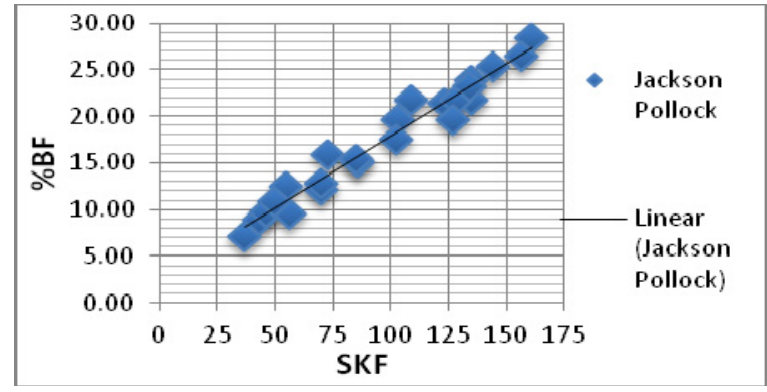

Figure 8. Jackson and Pollock $r=0.981 \mathrm{JP}$

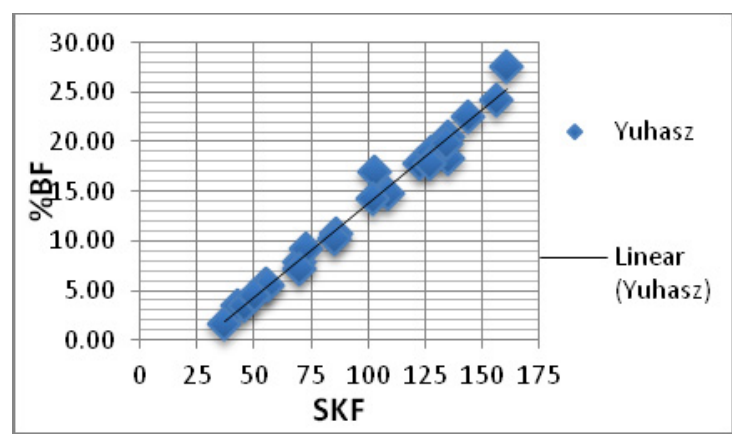

Figure 10. Yuhasz $r=0.992 \mathrm{Y}$

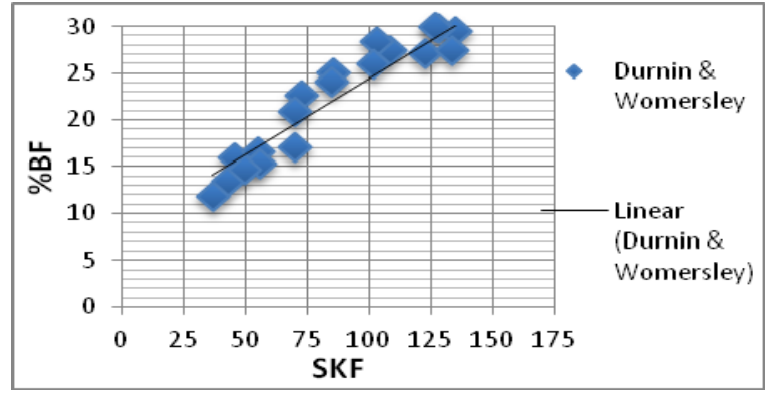

Figure 9. Durin Womersley $r=0.961 \mathrm{DW}$

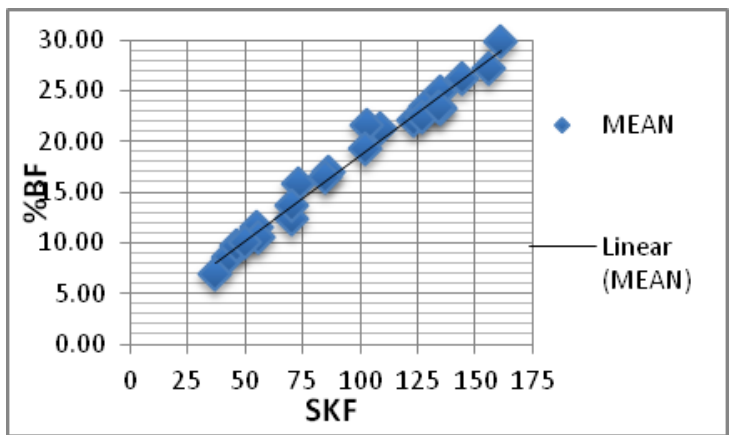

Figure 11. Mean Score $r=0.989$ MS 
The MS and Y displayed very high relationships of significance for females, acknowledging their reliability relationship of significance of SKF to \%BF.

\section{Discussion}

How do we interpret the BMI digital diagrams and what do the results really tell us in reference to well-trained full time athletes in recording and measurement of their body composition? And how can this assist in reliability of future comparisons? Digital diagrams $1 \& 2$ express concerns especially for 5 of the athlete scores - 3 Underweight, 2 overweight and additionally another 11 athlete scores at the high end of normal nearing overweight.

Are there significant changes to be made for these athletes and sending them to medical personnel and dietitians to address these concerns? As being categorised anorexia and obese are serious health issues. However before making any conclusions, it is important to compare the data of predicted $\% \mathrm{BF}$ and to look at the athletes who were tested at both periods. Table 6 illustrates you can measure improvement gains of LBM (less \%BF) whilst maintaining bodyweight, which is critical for athletes in combat sports dependent on weight divisions and aiming for increases in power to bodyweight ratios. Whereas the BMI scores had little or no change suggesting I's inability to determine reliable measureable assessment of changes in body composition of well-trained athletes such as the subjects in this study analysis. N.B. The predicted \%BF of the subjects in the BMI ranges of anorexia, high range normal and obese had very suitable predicted \%BF from the MEAN of the 3 protocol equations, indicative for their chosen sport and division as set out in the tables $4 \& 5$. Deurenburg et al (Note 6), derived at a BMI prediction \%BF formulae with age and gender input being: BMI Adult Fat $\%=(1.20 \mathrm{x}$ BMI $)+$ $(0,23 \times$ Age $)-(10.8 \times$ SEX $)-5.4($ sex values - Male $=1$, Female $=0)$.

Still with this formulae the male and female mean BMI scores and mean age of this analysis have the well trained athletes predicted $\% \mathrm{BF}$ over estimated at $18.15 \%$ (Male) and $27.12 \%$ (Female)compared to the 3 Protocol SKF equations MS of predicted \%BF $10.91 \pm 3.94 \& 18.20 \pm 6.25$ respectively. Suggesting the use of $\mathrm{BMI}$ in an equation falls short of addressing the physical make up of a well-trained athlete regarding LBM. The hypothesis was the MS (of the 3 predictors) would reduce the variation in error of over or underestimating \%BF with the goal to link the relationship of the MS to the sum of the 8 SKF measures establishing estimation guideline parameters of SKF SUM to \% BF. There will always be concerns expressing the difficulties of accurate recordings of SKF measures due to human error, calibration errors of calipers and SEE of the BF prediction equations. The key is to regularly conduct measurements to minimize inaccuracies in the tester and record the data to enable plotting a trend-line of the recordings to indicate the movement trends of the athletes SKF SUM linked to predicted $\%$ BF guidelines. Graphs $1 \& 2$ details the MEAN of each predicted \% BF equation and the MS of the 3 equations for both male and female. As noted earlier from Eston RG et al (1995), Jackson and Pollock and Durnin and Womersley equations over and underestimated predicted \%BF as compared to the MS respectively for both male and female. The Yuhasz equation for males revealed to be the most consistent in results of predicted $\% \mathrm{BF}$ of well-trained athletes producing the lowest of all STDEV of just 3.16 with just $0.65 \%$ difference in $\mathrm{BF}$ to the overall MS.

Indicating this to be a suitable equation for athletic males, Candido et al (Note 7) also supports this stating "The Yuhasz equation may be a good option for estimating F\% in young, physically trained men". However the Yuhasz equation did not have the same impact for females indicating quite the reverse with a raw \%BF range from $1.61 \%$ to $27.66 \%$ for the 23 SKF calculations and having the highest STDEV of 7.50 , indicating the Yuhasz equation has difficulty in consistency in the prediction of $\% \mathrm{BF}$ in athletic females. Male predicted $\% \mathrm{BF}$ means of each equation compared to the MS was relatively small in variance suggesting you could use these 3 equations as suitable field predictors of \%BF for well-trained male athletes with the mean of all three to be more reliable. If you were to use just one equation because of the low STDEV of the Yuhasz SKF Test of 3.16 as opposed to STDEV of 5.11 JP and 5.56 DW the Yuhasz equation for well-trained male athletes should produce a more consistent $\% \mathrm{BF}$ prediction with less \pm variation. However the 3 protocol equations used in this study for prediction $\% \mathrm{BF}$ for well-trained athletic males can be considered as a worthy measure for future data recordings and assessment. Other research articles such as Moon et al 2009 and Loenneke et al 2013 offer further support to the Jackson and Pollock and Durnin and Womersly equations as appropriate estimators for field-testing compared with reliability and validity of BIA and 4 component body fat prediction. The female equations displayed some different variations with a high \% under and over estimation of $\% \mathrm{BF}$ for the $\mathrm{Y}$ and $\mathrm{DW}$ equations whereas the JP 3 site equation produced more reliable and consistent prediction of $\% \mathrm{BF}$, very close to the MS. Reviewing the individual raw data predicted \%BF of the $\mathrm{Y}$ equation for female subjects, 6 subjects were under 5.73\% BF (with $1 @ 1.61 \% \mathrm{BF}, 2 @ 3.56 \% \mathrm{BF}$ ) suggesting there are some deficiencies in predicting \%BF of athletic females as these 6 subject estimates were obvious standout errors, whereas the JP was a very close 
predictor of $\% \mathrm{BF}$ to the MS for athletic females.

In consideration of these findings for future SKF equation recordings of athletic female subjects if the protocol is to continue with 3 equations it may be appropriate to replace the Yuhasz equation with another prediction \%BF equation or alternatively the MS of the Jackson and Pollock and Durnin and Womersley equations may suffice.

\section{Conclusions}

\subsection{Correlation between the Sum of 8 Skinfold Range to Predicted \% Body Fat Range in Well-Trained Athletes}

An objective of the analysis was to seek a suitable method as a field test for prediction of \%BF to be used as a monitoring training tool for sports performance practitioners in addressing abnormalities in \%BF. It's understood there is a reasonable error \% in determining \%BF through anthropometric SKF's in field-testing with the use of calipers and human error. The findings of this study suggest there are encouraging correlation trends forming as detailed in tables 4 and 5 of the article with the use of recording the MS of 3 SKF equations to minimize error calculations and the data analysis displays trends linking the sum of 8 SKF measures to a predicted \%BF range. It is recommended the data sample should be enlarged before determining definitive conclusions. Therefore it would be advantageous to gather a larger database to validate credibility and reliance of a predicted $\% \mathrm{BF}$ range linked to the sum of 8 SKF range for future research.

\subsection{Excluding BMI as a Reliable Measure of Body Composition for Well-Trained Athletes}

This article supports other research studies that BMI is not an appropriate measure of body composition assessment for well-trained athletes and should not be used as thus for sports people as a performance measurable assessment protocol. This study analysis has displayed the inconsistency and inaccurate measures of the BMI method, indicating BMI can be misleading categorising a significantly high $\%$ of well-trained athletes into the end stages of normal, overweight or obese, whereas this is contradictory to the equation predictions of \% BF of SKF equations. Demonstrating BMI method gives no allowance for improvement of an athletes' reduction of body fat and an increase in muscular development (LBM) whilst maintaining body weight. Highlighting BMI has deficiencies in determining a true reflection of a well-trained athletes body composition.

\section{References}

Durnin, J. V., \& Womersley, J. (1974). Body fat assessed from total body density and its estimation from skinfold thickness: Measurements on 481 men and women aged from 16 to 72 years. The British journal of nutrition, 32(1). http://dx.doi.org/10.1079/BJN19740060

Eston, R. G., Fu, F., \& Fung, L. (1995). Validity of conventional anthropometric techniques for predicting body composition in healthy Chinese adults. Br. J. Sports Med., 29(1), 52-56. http://dx.doi.org/10.1136/bjsm.29.1.52

Jackson, A. S., \& Pollock, M. L. (1977). Prediction accuracy of body density, lean weight, and total body volume equations. Med. Sci. Sports Exercise, 9, 197-201.

Jackson, A. S., Pollock, M. L. (1978). Generalized equations for predicting body density of men. Br J Nutr., 40(3), 497-504. http://dx.doi.org/10.1079/BJN19780152

Jackson, A. S., Pollock, M. L., \& Ward, A. (1980). Generalized equations for predicting body density of women. Med Sci Sports Exerc., 12(3), 175-181. http://dx.doi.org/10.1249/00005768-198023000-00009

Loenneke, J. P., Barnes, J. T., Wilson, J. M., Lowery, R. P., Isaacs, M. N., \& Pujol, T. J. (2013, May 3). Reliability of field methods for estimating body fat. Clin Physiol Funct Imaging. http://dx.doi.org/10.1111/cpf.12045

Moon, J. R., Tobkin, S. E., Smith, A. E., Lockwood, C. M., Walter, A. A., Cramer, J. T., ... Stout, J. R. (2009). Anthropometric estimations of percent body fat in NCAA Division I female athletes: a 4-compartment model validation. J Strength Cond Res., 23(4), 1068-1076. http://dx.doi.org/10.1519/JSC.0b013e3181aalcd0

Skinfold, Y. (n. d.). Test 101 Performance Evaluation Tests by Brian Mackenzie.

\section{Notes}

Note 1. Scientific Psychic http://www.scientificpsychic.com

Note 2. Eknoyan G. Nephrol Dial Transplant. 2008 Jan; 23(1):47-51. Epub 2007 Sep 22.Adolphe Quetelet (1796-1874) - the average man and indices of obesity.

Note 3. Professor Brian Curless Brett Allen, Zoran Popović The space of human body shapes: reconstruction and 
parameterization from range scans. Digital Humans, - Departm33ent of Computer Science \& Engineering, University.

Note 4. Body Composition: Skin-fold Measurements http://www.-rohan.sdsu.edu

Note 5. Physical Performance Measurement Procedural Manual, by Robert Ballard for National Sports Committee Indonesia Province West Sumatera, Indonesia. 2012.

Note 6. Deurenberg P, Weststrate J. A., Seidell J.C. BMI as a measure of body fatness age and sex specific.

Note 7. Cândido Simões Pires Neto, Maria Fátima Glaner -The "faulkner equation" for predicting body fat: The end of a myth.

\section{Copyrights}

Copyright for this article is retained by the author(s), with first publication rights granted to the journal.

This is an open-access article distributed under the terms and conditions of the Creative Commons Attribution license (http://creativecommons.org/licenses/by/3.0/). 